\title{
Neratinib for the Treatment of Early- Stage HER2-Positive Breast Cancer
}

\author{
JENNIFER MILES, PharmD, and YAHSIN WHITE, PharmD
}

From Wellmont Cancer Institute-Johnson City, Johnson City, Tennessee

Authors' disclosures of conflicts of interest are found at the end of this article.

Correspondence to: Jennifer Miles, PharmD, 378 Marketplace Blvd, Suite 10, Johnson City, TN 37604.E-mail: jennifer.miles2@balladhealth.org https://doi.org/10.6004/jadpro.2018.9.7.6 (c) 2018 Harborside ${ }^{\text {TM }}$
J Adv Pract Oncol 2018;9(7):750-754

\begin{abstract}
The treatment of breast cancer has been revolutionized by the development of HER2-targeted treatments for patients who are HER2 positive. The HER2 protein is present at high levels in about $30 \%$ of breast cancer patients. These high levels are associated with a greater chance of metastasis, relapse, and decreased survival. The current standard of care for early-stage HER2-positive patients includes treatment with 1 year of trastuzumab therapy. Although trastuzumab has improved outcomes, there is still a $20 \%$ chance for tumor recurrence and a $16 \%$ chance of death. Neratinib was developed to give patients with earlystage HER2-positive breast cancer an option to increase the diseasefree survival rate. The 5 -year invasive disease-free survival rate was $90.2 \%$ (95\% confidence interval $=88.3-91.8)$ in the neratinib group and $87.7 \%$ (95\% confidence interval $=85.7-89.4)$ in the placebo group.
\end{abstract}

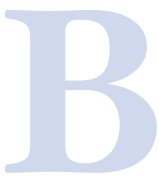

reast cancer is the most common type of cancer and the second leading cause of cancer death in women. The American Cancer Society (2018) estimates that about 266,120 new cases of invasive breast cancer and 63,960 new cases of carcinoma in situ will be diagnosed this year in the United States. It is estimated that 40,920 will die in 2018 of breast cancer. Human epidermal growth factor receptor 2 (HER2) plays a role in the prognosis and treatment of breast cancer (National Comprehensive Cancer Network, 2018). HER2 genes make receptors on breast cells. When working correctly, they control how healthy cells grow, divide, and repair themselves. In breast cancer, the HER2 gene does not work like it should and makes too many HER2 receptors. This in turn causes breast cells to grow and divide incorrectly and uncontrollably (Breastcancer.org, 2018). HER2 overexpression will be present in $20 \%$ to $30 \%$ of cases. HER2-positive breast cancer is often more aggressive, with a higher recurrence rate and decreased survival rate compared to other forms of breast cancer (Mitri, Constantine, \& O’Regan, 2012).

There are several breast cancer treatments available in the United States designed specifically to target HER2 receptors. They include trastuzumab (Herceptin), ado- 
trastuzumab emtansine (Kadcyla), pertuzumab (Perjeta), lapatinib (Tykerb), and neratinib (Nerlynx; National Comprehensive Cancer Network, 2018). Neratinib is an oral kinase inhibitor that was approved by the US Food and Drug Administration (FDA) in July 2017 for extended adjuvant treatment of adult patients with early-stage HER2-overexpressed/amplified breast cancer following adjuvant trastuzumab-based therapy (Puma Biotechnology Inc., 2017).

\section{PHARMACOLOGY AND PHARMACOKINETICS}

Neratinib irreversibly binds to several epidermal growth factor receptors (EGFR) that include EGFR, HER2, and HER4. Neratinib reduces EGFR and HER2 autophosphorylation, which blocks downstream signaling pathways and exhibits antitumor activity in EGFR- and HER2-expressing carcinoma cell lines. Autophosphorylation is a process in which a phosphate is added to a protein kinase by itself. It takes place by the addition of a phosphate group to serine, threonine, or tyrosine residues in protein kinases (Puma Biotechnology Inc., 2017).

Neratinib is primarily metabolized by the hepatic enzyme CYP3A4. Its half-life ranges from 7 to 17 hours, and is mostly excreted through feces. The drug's time to peak effect is 2 to 8 hours after consumption. Absorption is increased by a highfat meal and is affected by gastric $\mathrm{pH}$ (Lexicomp Online, 2018).

\section{CLINICAL EFFICACY}

Approval for neratinib was based on the results of the ExteNET trial, which was a multicenter, randomized, double-blind, placebo-controlled, phase III trial that compared 1 year of treatment with neratinib vs. placebo in patients with HER2positive breast cancer in patients who had already received trastuzumab for 1 year. The women aged 18 years or greater had stage I through III HER2positive breast cancer and had completed neoadjuvant and adjuvant trastuzumab therapy up to 2 years before randomization. Later, the inclusion criteria changed to include patients with stage II to III HER2-positive breast cancer who had completed trastuzumab therapy up to 1 year previously. There were 2,840 patients randomly assigned to receive either neratinib at $240 \mathrm{mg}$ per day ( $\mathrm{n}=$
$1,420)$ or placebo $(n=1,420)$. Randomization was stratified by hormone receptor status, nodule status, and whether trastuzumab was given sequentially vs. concurrently with other chemotherapy. The major efficacy outcome measure was invasive disease-free survival (iDFS), defined as the time between the dates of randomization to the first occurrence of invasive recurrence (local/regional, ipsilateral, or contralateral breast cancer), distant recurrence, or death from any cause within 2 years and 28 days of follow-up (Chan et al., 2016).

Median follow-up was 24 months in both groups. There were 67 iDFS events in patients receiving neratinib and 106 events in the placebo group (hazard ratio [HR], 0.66; 95\% confidence interval $[\mathrm{CI}]=0.49-0.90 ; p=.008)$. The 2 -year iDFS rate was $94.2 \%(\mathrm{CI}=92.6-95.4)$ in the neratinib group and $91.9 \%(\mathrm{CI}=90.2-93.2)$ in the placebo group (Puma Biotechnology Inc., 2017).

Updated efficacy outcomes were reported from a protocol-defined 5-year follow-up analysis. After a median follow-up period of 5.2 years, there were 116 iDFS events in the treatment group vs. 163 events in the placebo group (HR, 0.73; CI $=0.57-0.92 ; p=.008)$. The 5 -year iDFS rate was $90.2 \%(\mathrm{CI}=88.3-91.8)$ in the neratinib group and $87.7 \%(\mathrm{CI}=85.7-89.4)$ in the placebo group (Martin et al., 2017).

\section{ADVERSE DRUG REACTIONS}

In clinical trials, the most common adverse reactions (> 5\%) reported with neratinib were fatigue, rash, stomatitis, muscle spasms, aspartate aminotransferase or alanine aminotransferase increase, nail disorder, dry skin, weight loss, nausea, urinary tract infection, diarrhea, vomiting, decreased appetite, dyspepsia, and abdominal distention. Diarrhea, vomiting, nausea, and abdominal pain were the most frequently reported grade 3 and 4 adverse reactions. Dose reduction due to an adverse reaction occurred in $31.2 \%$ of patients receiving neratinib vs. $2.6 \%$ of patients receiving placebo. Discontinuation of neratinib due to any adverse reaction was reported in $27.6 \%$ of treatment patients, and $16.8 \%$ of those patients discontinued due to diarrhea (Puma Biotechnology Inc., 2017).

Diarrhea is the most common toxicity associated with neratinib, but it can be effectively managed with early intervention. Safety data suggest 
that using intensive antidiarrheal prophylaxis reduces the severity and duration of diarrhea. It is recommended to initiate loperamide with the first dose of neratinib and continue during the first two cycles (Puma Biotechnology Inc., 2017).

\section{DOSING AND ADMINISTRATION}

The dosing recommendation is $240 \mathrm{mg}$ orally (six 40-mg tablets) once a day to be taken with food continuously for 12 months. Patients should take neratinib whole (not split, chewed, or crushed) at the same time every day. If the patient forgets a dose, the patient should be advised to skip that dose and resume the neratinib the next day at the same scheduled time. The starting dose of neratinib needs to be reduced to $80 \mathrm{mg}$ once a day in patients with severe hepatic impairment (ChildPugh class C; Puma Biotechnology Inc., 2017).

Dose interruptions and reductions may be made if needed for individual patient safety and tolerance to neratinib. Because neratinib is primarily metabolized through the liver, treatment interruption and dose adjustments should be made if hepatic toxicity occurs during treatment. Hold neratinib for any grade 3 toxicity until recovery to grade 1 or baseline and then restart neratinib at a lower dose. Table 1 shows dose modifications for adverse reactions. For any grade 4 toxicities, discontinue neratinib permanently (Puma Biotechnology Inc., 2017).

Loperamide prophylaxis is recommended for the first 56 days ( 2 cycles) of neratinib to prevent diarrhea. As shown in Table 2, loperamide at $4 \mathrm{mg}$ can be taken orally three times daily during days 1 through 14 of treatment, then decreased to twice daily on days 15 through 56 of treatment. Patients can take loperamide orally as needed up to $16 \mathrm{mg}$ (4 tablets) per day for the remainder of their treatment to maintain one to two bowel movements per day (Puma Biotechnology Inc., 2017).
The CONTROL trial is a phase II study currently looking at the effects of adding budesonide or colestipol to loperamide prophylaxis for neratinib-associated diarrhea. For the first cycle of neratinib, patients received either budesonide at $9 \mathrm{mg}$ extended release once a day or colestipol at $2 \mathrm{~g}$ twice a day in addition to the recommended loperamide treatment schedule. Preliminary data indicates prophylaxis with colestipol plus loperamide resulted in fewer discontinuations due to diarrhea (1.7\%) compared to budesonide plus loperamide (10.9\%) and loperamide alone (20.4\%). The colestipol plus loperamide group also had fewer treatment interruptions (9.2\%) and treatment reductions $(4.2 \%)$ compared to loperamide alone ( $15.3 \%$ and $7.3 \%$, respectively). The trial is ongoing and the final analysis will be performed when all patients have completed 12 months of neratinib therapy (Hurvitz et al., 2017).

\section{IMPLICATIONS FOR THE AP}

It is important to provide thorough patient counseling and education for patients prescribed neratinib. Patients should be advised on the occurrence and possible severity of diarrhea while taking this medication. Patients need to be given written instructions for loperamide prophylaxis to help reduce episodes of diarrhea. Current labeling does not include recommendations to add budesonide or colestipol for diarrhea prevention; however, patients may benefit from using dual therapy. Patients need to be instructed to notify their provider immediately if they are experiencing severe diarrhea ( $\geq 7$ stools per day) or diarrhea with weakness, dizziness, or fever. Patients should increase fluid intake to 2 liters a day to avoid dehydration. Dietary modifications may be necessary if loperamide does not work well enough to reduce the diarrhea. Clinicians should explain to the patient

Table 1. Neratinib Dose Modifications for Adverse Reactions

\section{Dose level}

Recommended starting dose

First dose reduction

Second dose reduction

Third dose reduction

\section{Neratinib dose}

$240 \mathrm{mg}$ (6 tablets) daily

200 mg (5 tablets) daily

$160 \mathrm{mg}$ (4 tablets) daily

$120 \mathrm{mg}$ (3 tablets) daily

Note. Information from Puma Biotechnology Inc. (2017). 


\section{Table 2. Loperamide prophylaxis}

\section{Time on neratinib}

Weeks 1-2 (days 1-14)

Weeks 3-8 (days 15-56)

Weeks 9-52 (days 57-365)
Loperamide dose

$4 \mathrm{mg}$

$4 \mathrm{mg}$

$4 \mathrm{mg}$

\section{Frequency}

Three times daily

Two times daily

As needed (up to $16 \mathrm{mg}$ per day)

Note. Information from Puma Biotechnology Inc. (2017).

that sometimes dose interruptions and reductions may need to be made to reduce diarrhea and sometimes neratinib will have to be discontinued if diarrhea cannot be controlled. Table 3 illustrates how to manage diarrhea with neratinib.

Patients need to be informed of the potential for hepatotoxicity when taking neratinib. It is suggested that blood tests be performed before treatment is started, monthly for the first 3 months, then every 3 months thereafter. Patients need to be instructed to notify their provider if symptoms of liver dysfunction such as tiredness, nausea, vomiting, abdominal pain, fever, rash, itching, or yellowing of the skin or eyes occur during treatment. As shown in Table 4, dose interruptions and reductions can also occur with hepatic toxicity as well (Puma Biotechnology Inc., 2017).
Female patients need to have a pregnancy test before starting treatment with neratinib and be instructed to take birth control during treatment and for at least 1 month after their last dose. For male patients, female partners need to be on a form of birth control for 3 months after treatment (Puma Biotechnology Inc., 2017).

It is important to discuss all medications that the patient is receiving and the importance of telling the health-care team of any new medications, including over-the-counter and herbal supplements. It is particularly important to discuss avoiding medications that are gastric acid-reducing, such as $\mathrm{H}_{2}$-receptor antagonists and proton pump inhibitors, as concomitant use with these medications can result in a decrease of neratinib $\mathrm{C}_{\max }$ by $71 \%$ and area under the curve (AUC) by

\section{Table 3. Dose Modifications for Diarrhea}

\section{Severity of diarrhea} baseline)

Grade 2 diarrhea (increase of 4-6 stools per day over baseline) lasting $<5$ days

Grade 3 diarrhea (increase of $\geq 7$ stools per day over baseline; incontinence; hospitalization indicated; limiting self-care activities of daily living) lasting $<2$ days

Any grade with complicated features such as dehydration, fever, hypotension, renal failure, or grade 3 or 4 neutropenia

Grade 2 diarrhea lasting 5 days or longer despite being treated with optimal medical therapy

Grade 4 diarrhea (life-threatening consequences; urgent intervention indicated)

Diarrhea recurs to grade 2 or higher at $120 \mathrm{mg}$ of neratinib per day
Grade 1 diarrhea (increase of $<4$ stools per day over

\section{Action}

- Adjust antidiarrheal treatment

- Diet modifications

- Fluid intake of around $2 \mathrm{~L}$ should be maintained to avoid dehydration

- Once event resolves to $\leq$ grade 1 or baseline, start loperamide at $4 \mathrm{mg}$ with each subsequent neratinib administration

- Interrupt neratinib treatment

- Diet modifications

- Fluid intake of around $2 \mathrm{~L}$ should be maintained to avoid dehydration

- If diarrhea resolves to grade 0-1 in 1 week or less, then resume neratinib treatment at the same dose

- If diarrhea resolves to grade 0-1 in longer than 1 week, then resume neratinib treatment at reduced dose (see Table 1)

- Once event resolves to $\leq$ grade 1 or baseline, start loperamide at $4 \mathrm{mg}$ with each subsequent neratinib administration

- Permanently discontinue neratinib treatment

- Permanently discontinue neratinib treatment

Note. Information from Puma Biotechnology Inc. (2017). 
Table 4. Dose Modifications for Hepatotoxicity

\begin{tabular}{|c|c|}
\hline Severity of hepatotoxicity & Action \\
\hline $\begin{array}{l}\text { Grade } 3 \text { ALT (> 5x-20x ULN) } \\
\text { OR } \\
\text { Grade } 3 \text { bilirubin ( }>3 x-10 x \text { ULN) }\end{array}$ & $\begin{array}{l}\text { - Hold neratinib until recovery to } \leq \text { grade } 1 \\
\text { - Evaluate alternative causes } \\
\text { - Resume neratinib at the next lower dose level if recovery to } \leq \text { grade } 1 \text { occurs } \\
\text { within } 3 \text { weeks. If grade } 3 \text { ALT or bilirubin occurs again despite one dose } \\
\text { reduction, permanently discontinue neratinib }\end{array}$ \\
\hline $\begin{array}{l}\text { Grade } 4 \text { ALT (> 20x ULN) } \\
\text { OR } \\
\text { Grade } 4 \text { bilirubin (> 10x ULN) }\end{array}$ & $\begin{array}{l}\text { - Permanently discontinue neratinib } \\
\text { - Evaluate alternative causes }\end{array}$ \\
\hline
\end{tabular}

$65 \%$. If patients must use antacids, they should avoid taking antacids within 3 hours of the neratinib. Because neratinib is primarily metabolized through CYP3A4, strong or moderate CYP3A4 inhibitors and inducers also need to be avoided when taking neratinib. CYP3A4 inhibitors have the potential to increase the concentration of neratinib and increase the risk of toxicity. CYP3A4 inducers decrease the concentration of neratinib and lower its effectiveness by lowering the AUC (Puma Biotechnology Inc., 2017).

\section{CONCLUSION}

Neratinib has been shown to be an effective adjuvant treatment for early-stage HER2-postive breast cancer following adjuvant therapy with trastuzumab. The dosing recommendation is $240 \mathrm{mg}$ orally (six 40-mg tablets) once a day to be taken with food continuously for 12 months. The cost of neratinib is $\$ 10,400$ for a 30 -day supply. The manufacturer offers commercially insured, eligible patients a copay assistance card to help with out-of-pocket costs (nerlynx.com/access-and-support).

The ExteNET trial showed statistically significant results in 5-year survival rates in women treated with neratinib vs. placebo. Patients need to be monitored for potential severe adverse reactions. Ensuring the patient has a good understanding of the preventive care available can help reduce some of the adverse effects associated with neratinib. Neratinib provides a good option for practitioners to offer to their patients (Chan et al., 2016).

\section{Disclosure}

The authors have no conflicts of interest to disclose.

\section{References}

American Cancer Society. (2018). Cancer facts \& figures 2018. Retrieved from https://www.cancer.org/content/dam/ cancer-org/research/cancer-facts-and-statistics/annual-cancer-facts-and-figures/2018/cancer-facts-and-figures-2018.pdf

Breastcancer.org. (2018). HER2 status. Retrieved from http://www.breastcancer.org/symptoms/diagnosis/ her2

Chan, A., Delaloge, S., Holmes, F. A., Moy, B., Iwata, H., Harvey, V. J.,...Martin, M. (2016). Neratinib after trastuzumabbased adjuvant therapy in patients with HER2-positive breast cancer (ExteNET): A multicentre, randomised, double-blind, placebo-controlled phase 3 trial. Lancet Oncology, 17(3), 367-377. https://doi.org/10.1016/S14702045(15)00551-3

Hurvitz, S., Chan, A., Iannotti, N., Ibrahim, E., Chien, J., Chan, N.,...Rugo, H. S. (2017). Effects of adding budesonide or colestipol to loperamide prophylaxis on neratinib-associated diarrhea in patients with HER2+ early-stage breast cancer: The CONTROL trial. Poster presented at the 2017 San Antonio Breast Cancer Symposium. Retrieved from http://www.pumabiotechnology.com/docs/P3-14-01120517PBYIatSABCSCONTROLPoster.pdf

Lexicomp Online. (2018). Neratinib. Retrieved from https:// online.lexi.com/lco/action/doc/retrieve/docid/ patch_f/6519378

Martin, M., Holmes, F. A., Ejlertsen, B., Delaloge, S., Iwata, H., von Minckwitz, G.,...Ciceniene, A. (2017). Neratinib after trastuzumab-based adjuvant therapy in HER2positive breast cancer (ExteNET): 5-year analysis of a randomised, double-blind, placebo-controlled, phase 3 trial. Lancet Oncology, 18(12), 1688-1700. https://doi. org/10.1016/S1470-2045(17)30717-9

Mitri, Z., Constantine, T., \& O'Regan, R. (2012). The HER2 receptor in breast cancer: Pathophysiology, clincal use, and new advances in therapy. Chemotherapy Research and Practice, 2012. https://doi.org/10.1155/2012/743193

National Comprehensive Cancer Network. (2018). NCCN Clinical Practice Guidelines in Oncology: Breast Cancer. v1.2018. Retrieved from https://www.nccn.org/professionals/physician_gls/pdf/breast.pdf

Puma Biotechnology Inc. (2017). Nerlynx (neratinib) package insert. Retrieved from https://www.accessdata.fda.gov/ drugsatfda_docs/label/2017/208051s000lbl.pdf 